\title{
Lectin and immunohistochemical comparison of glycoconjugates in the conjunctiva of patients with and without exfoliation syndrome
}

\author{
Jaana Hietanen, Marita Uusitalo, Ahti Tarkkanen, Tero Kivelä
}

\begin{abstract}
Aims-The study was carried out to search for labelling similar to that of intraocular exfoliation material in the conjunctiva by light microscopy using lectin and immunohistochemistry.

Methods-Ten formalin fixed and paraffin embedded conjunctival biopsy specimens both from patients with and without exfoliation syndrome were studied with a panel of 11 lectins and with three monoclonal antibodies to the HNK-1 carbohydrate epitope, all of which react with intraocular exfoliation material.
\end{abstract}

Results-The lectin binding profile was essentially the same in specimens from patients with and without exfoliation syndrome. The superficial epithelium reacted similarly with Phaseolus vulgaris (PHA-E), Caragana arborescens (CAA), Helix pomatia (HPA), concanavalin A (ConA), and wheat germ (WGA) agglutinins. Binding was also detected with peanut (PNA) and Bauhinia purpurea (BPA) agglutinins, particularly in patients with exfoliation. The basement membrane generally reacted with Ricinus communis (RCA-I), PHA-E, Vicia villosa (VVA), ConA, and Lens culinaris (LCA) agglutinins. The stroma was weakly labelled with RCA-I, PHA-E, ConA, and LCA. Lectin binding to the vascular endothelium was moderate with RCA-I, PHA-E, CAA, ConA, LCA, and WGA. Inconsistent labelling was also detected with PNA, BPA, and Erythrina cristagalli agglutinin (ECA). The subendothelial region reacted weakly but consistently with PHA-E, ConA, and LCA, and inconsistently with PNA. Pretreatment with neuraminidase did not change that pattern. Antibodies to the HNK-1 epitope reacted only with myelinated stromal nerve branches.

Conclusion-No evidence of abnormal deposits in any specimen was found. The carbohydrate composition of intraocular exfoliation material may differ from that of exfoliation-like fibres often detected in the conjunctiva by electron microscopy. (Br f Ophthalmol 1995; 79: 467-472)

Exfoliation syndrome has traditionally been considered an intraocular disorder. ${ }^{1}$ Several electron microscopic studies have, however, reported exfoliation-like fibres around blood vessels and in the stroma of the conjunctiva in patients with exfoliation syndrome. ${ }^{2-8}$ Additionally such fibres have been noted in other extraocular structures, including skin, heart, lung, liver, kidney, and meninges. ${ }^{9-12}$ The identification of extrabulbar exfoliationlike fibres has been based on morphology, and variation in their structure has been detected. ${ }^{7-9}$

Lectin and immunohistochemical studies have shown that, in addition to basement membrane components, ${ }^{13} 14$ intraocular exfoliation material reacts consistently with several lectins recognising various sugar residues and with antibodies to the HNK-1 carbohydrate epitope containing a 3-sulphoglucuronic acid residue. ${ }^{15-18}$ To screen for these glycoconjugates in the conjunctiva of patients with and without exfoliation syndrome, a panel of lectins and three antibodies to the HNK-1 epitope were used that all bind to exfoliation material. ${ }^{15-19}$ Since conventional light microscopic studies have failed to detect exfoliation deposits in the conjunctiva, evidence of labelling similar to intraocular exfoliation material was searched for using lectin and immunohistochemistry as additional tools. Such methods would be more easy and rapid to apply for screening multiple biopsies from large study populations than electron microscopy.

\section{Material and methods}

HISTOLOGICAL SPECIMENS

Conjunctival biopsy specimens of approximately $2 \times 4 \mathrm{~mm}$ in size were taken from the upper limbal region during cataract surgery. The study was approved by the local ethics committee. Ten patients (eight women and two men, age range $60-87$ years, mean age 76 years) had typical exfoliation material in the anterior segment of the eye by preoperative slit-lamp examination. Three of them had capsular glaucoma, one had ocular hypertension, one had been treated previously because of acute angle closure glaucoma, and five had exfoliation syndrome only. Additionally, similar biopsies from 10 patients (eight women and two men, age range 52 to 90 years, mean age 77 years) without any clinical evidence of exfoliation material in either eye were chosen for controls. Exfoliation material in five enucleated or autopsy eyes with capsular glaucoma, and goblet cells of the conjunctival specimens were stained as positive controls for binding of lectins and antibodies to the HNK-1 epitope in the present study. ${ }^{16-18} 20$ 
Table 1 Characteristics of the 11 lectins used *

\begin{tabular}{|c|c|c|c|}
\hline Lectin (abbreviation) & Nominal carbohydrate specificity & Inhibitor & $\begin{array}{l}\text { Reactivity with } \\
\text { exfoliation material }\end{array}$ \\
\hline $\begin{array}{l}\text { Arachis hypogaea (PNA) } \\
\text { Bauhinia purpurea alba (BPA) } \\
\text { Canavalia ensiformis (ConA) } \\
\text { Caragana arborescens (CAA) } \\
\text { Erythrina cristagalli (ECA) } \\
\text { Helix pomatia (HPA) } \\
\text { Lens culinaris (LCA) } \\
\text { Phaseolus vulgaris (PHA-E) } \\
\text { Ricinus communis (RCA-I) } \\
\text { Triticum vulgaris (WGA) } \\
\text { Vicia villosa (VVA) }\end{array}$ & $\begin{array}{l}\text { DGal }(\beta 1 \rightarrow 3) \text { DGalNAc }>\alpha / \beta D G a l \\
\alpha / \beta D G a l N A c>\alpha / \beta D G a l \\
\alpha-D-M a n>\alpha-D-G l c \\
\alpha / \beta D G a l N A c \\
\text { DGal }(\beta 1 \rightarrow 4) \text { DGlcNAc }>\alpha D G \text { alNAc } \\
\text { DGalNAc }(\alpha 1 \rightarrow 3) \text { DGalNAc }>\text { DGalNAc } \\
\alpha-D-M a n>\alpha-D-G l c \\
\text { DGal }(\beta 1 \rightarrow 4) \text { DGlcNAc }(\beta 1 \rightarrow 2) \text { DMan } \\
\beta D G a l>\alpha \text { DGal }>\alpha D G a l N A c \\
(\beta-D-G l c N A c), \text { NeuNAc } \\
\alpha \text { DGalNAc }>\alpha D G a l\end{array}$ & $\begin{array}{l}\text { DGal } \\
\text { DGal } \\
\alpha-M e t-D-M a n \\
\text { DGalNAc } \\
\text { DGalNAc } \\
\text { DGalNAc } \\
\alpha \text {-Met-D-Man } \\
\text { Fetuin } \\
\text { Lactose } \\
\text { DGalNAc } \\
\text { DGalNAc }\end{array}$ & $\begin{array}{l}\text { Strong } \\
\text { Strong } \\
\text { Strong } \\
\text { Weak } \\
\text { Weak } \\
\text { Moderate } \\
\text { Strong } \\
\text { Strong } \\
\text { Strong } \\
\text { Strong } \\
\text { Weak }\end{array}$ \\
\hline
\end{tabular}

^Modified from Goldstein and Poretz. ${ }^{26}$

$+\mathrm{Gal}=$ galactose, Man=mannose, GalNAc $=N$-acetylgalactosamine, $\mathrm{GlcNAc}=N$-acetylglucosamine, Met $=$ methyl derivative of sugar.

$\ddagger$ Hietanen and Tarkkanen ${ }^{16}$; Hietanen et al. ${ }^{18}$

All specimens were immediately fixed in formalin for 2 days and embedded in paraffin. Sections ( $5 \mu \mathrm{m}$ thick) were cut from the specimens and mounted on chromium-gelatin treated glass slides to ensure tissue adherence (0.05 $\mathrm{g}$ potassium chromium (III) sulphate dodecahydrate and $0.5 \mathrm{~g}$ gelatin in $100 \mathrm{ml}$ distilled water). Routine staining was with haematoxylin and eosin.

\section{LECTIN HISTOCHEMISTRY}

Sections were stained by lectin histochemistry using a commercial version (Vectastain ABC Elite Kit, Vector Laboratories, Burlingame, CA, USA) of the avidin-biotinylated peroxidase complex (ABC) method. ${ }^{21-23}$ Duplicate series were treated either with pepsin to enhance the availability of lectin binding sites in formalin fixed and paraffin embedded material, ${ }^{24}$ or with neuraminidase to expose penultimate carbohydrate residues blocked by sialic acid. ${ }^{25}$ Additionally, parallel control sections with corresponding hapten sugar inhibitors were stained (Table 1). The exact staining procedure has been explained in detail previously. ${ }^{18}$

Agglutinins (Table 1) ${ }^{26}$ from Arachis hypogaea (peanut agglutinin, PNA, lot $18 \mathrm{~F}-$ 40511), Bauhinia purpurea alba (BPA, lot 27F-4021), Canavalia ensiformis (ConA, concanavalin A, lot 49F-8030), Caragana arborescens (CAA, lot 21H-9550), Erythrina cristagalli (ECA, lot 56F-4018), Helix pomatia (HPA, lot 102H-38861), Lens culinaris (LCA, lot $61 \mathrm{H}-3903$ ), Phaseolus vulgaris (erythroagglutinin, PHA-E, type III-B, lot 77F-4012), Ricinus communis (agglutinin I, RCA-120, lot 20H-4051), Triticum vulgaris (WGA, wheat germ agglutinin, lot $111 \mathrm{H}-4016$ ), and Vicia villosa (VVA, lot 49F-8025) conjugated to biotin were commercially obtained from Sigma (St Louis, MO, USA) diluted with phosphate buffered saline (PBS) to a protein concentration of $500 \mu \mathrm{g} / \mathrm{ml}$, and stored at $-20^{\circ} \mathrm{C}$ until needed.

\section{IMMUNOHISTOCHEMICAL STAINING}

Sections were immunostained using a commercial version of the $\mathrm{ABC}$ method (Vectastain ABC Elite Kit for Mouse IgG, Vector Laboratories, Burlingame, CA, USA), as already described in detail. ${ }^{27}$ The primary IgM mouse monoclonal antibodies (MAbs) to the HNK-1 epitope used were NC-1 (CD57, IgM, lot 2204-01-02, Centraal laboratorium van de bloedtransfusiedienst, Amsterdam; 1:25 dilution), ${ }^{28}$ HNK-1 (Leu-7, IgM, lot 30015, Becton Dickinson, San Jose, CA, USA; 1:40 dilution), ${ }^{29}$ and VC1.1 (IgM, lot 071H4828, Sigma, St Louis, MO, USA; 1:16000 dilution). ${ }^{30}$

An unrelated IgM MAb CK B1 to cytokeratin 14 (lot 100H4800, Sigma, 1:300 dilution) $)^{31}$ was used as a negative control.

Table 2 Binding of lectins and MAbs to the HNK-1 epitope to the conjunctiva of patients with and without exfoliation syndrome*

\begin{tabular}{|c|c|c|c|c|c|c|}
\hline \multirow[b]{2}{*}{ Reagent } & \multicolumn{4}{|l|}{ Conjunctiva } & \multicolumn{2}{|c|}{ Conjunctival blood vessels } \\
\hline & Epithelium & $\begin{array}{l}\text { Basement } \\
\text { membrane }\end{array}$ & Stroma & $\begin{array}{l}\text { Goblet } \\
\text { cells }\end{array}$ & Endothelium & $\begin{array}{l}\text { Subendothelial } \\
\text { region }\end{array}$ \\
\hline PNA & $3 / 1$ & - & $-\dagger$ & $5 / 4 \ddagger$ & $3 / 1$ & $3 / 1$ \\
\hline BPA & $9 / 2 \dagger$ & - & - & $9 / 9^{\circ}$ & $6 / 9$ & - \\
\hline RCA-I & - & $9 / 10$ & $9 / 10$ & $4 / 7$ & $9 / 8$ & - \\
\hline ECA & - & - & $2 / 1$ & - & $2 / 5$ & _- \\
\hline PHA-E & $9 / 10$ & $7 / 9$ & $9 / 10$ & - & $9 / 10$ & $10 / 10$ \\
\hline CAA & $9 / 10$ & - & $3 / 4$ & $0 / 3$ & $9 / 10$ & $3 / 2$ \\
\hline VVA & - & $7 / 7$ & $7 / 9$ & - & $2 / 3$ & - \\
\hline HPA & $4 / 4 \dagger$ & - & - & $8 / 8$ & $2 / 2$ & - \\
\hline ConA & $10 / 10$ & $10 / 10$ & $10 / 9$ & - & $10 / 10$ & $10 / 9$ \\
\hline LCA & - & $10 / 9$ & $10 / 10$ & - & $9 / 10$ & $10 / 10$ \\
\hline WGA & $3 / 3 \ddagger$ & - & $2 / 2$ & $2 / 3 \ddagger$ & $3 / 3$ & $2 / 2$ \\
\hline NC-1 & - & - & $10 / 10 \$$ & - & - & - \\
\hline HNK-1 & - & - & $10 / 10 \S$ & - & - & - \\
\hline VC1·1 & - & - & $10 / 10 \S$ & - & - & - \\
\hline
\end{tabular}

*Number of positive specimens out of 10 in patients with/without exfoliation, $-=$ no reaction in any specimen studied. Owing to lack of material the number of specimens studied with PNA and WGA were 8 and 6, respectively.

†Enhanced by neuraminidase treatment.

¥Reduced by neuraminidase treatment.

Only myelinated stromal nerve branches were reactive. 


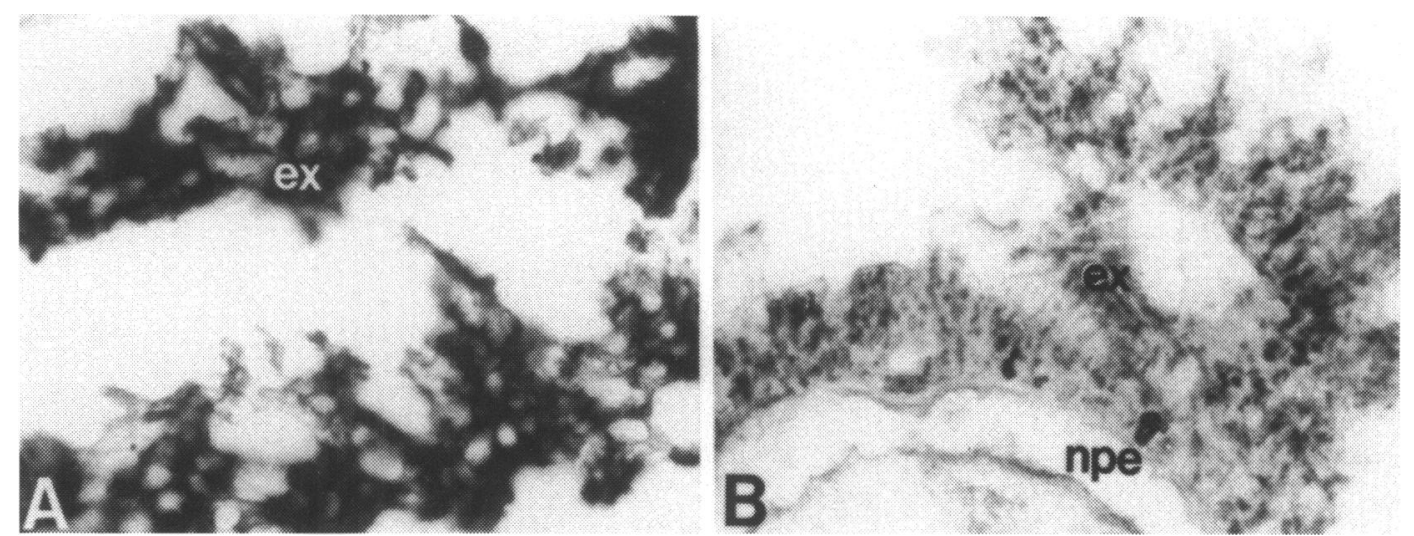

Figure 1 BPA reacts strongly with intraocular exfoliation material (ex) (A; lectin staining; $\times 500)$, which is also labelled with $M A B H N K-1$ on the detached, non-pigmented ciliary epithelium (npe) (B; immunoperoxidase staining; $\times 500)$.

Omission of the secondary antibody or the ABC complex caused loss of immunoreaction.

\section{Results}

Lectin binding to the conjunctiva and its immunoreactivity for the HNK-1 carbohydrate epitope are summarised in Table 2 . With few exceptions no differences at all were noted in the lectin binding profile of specimens with exfoliation compared with the controls showing no exfoliation. The binding of all lectins was abolished or greatly reduced after preincubation with their appropriate hapten sugar inhibitors. Intraocular exfoliation material reacted with lectins (Fig 1A) and antibodies to the HNK-1 carbohydrate epitope (Fig 1B) used in the present study.

\section{LECTIN HISTOCHEMISTRY}

The superficial conjunctival epithelium reacted consistently with Phaseolus vulgaris (PHA-E) (Fig 2A, B), Caragana arborescens (CAA), Helix pomatia (HPA), concanavalin A (ConA) (Fig 2C, D), and wheat germ (WGA) agglutinin in patients with and without exfoliation. Positive labelling was also detected with peanut (PNA) and Bauhinia purpurea (BPA) agglutinin, particularly in patients with exfoliation (Table 2 ). The binding of PNA, CAA, and WGA to the superficial epithelium was generally strong. The binding of PHA-E (Fig 2A, B) and ConA (Fig 2C, D) was moderate, and that of HPA weak. Pretreatment with neuraminidase clearly enhanced the binding of BPA and HPA, and decreased that of WGA.

The basement membrane of the epithelium reacted consistently with Ricinus communis (RCA-I), PHA-E (Fig 2A, B), Vicia villosa (VVA), ConA (Fig 2D), and Lens culinaris (LCA) agglutinins both in patients with and without exfoliation. The binding intensity was generally moderate. Pretreatment with neuraminidase had no influence on the lectin binding to the basement membrane.

The goblet cells reacted consistently with PNA, BPA (Fig 2E, F), HPA, and WGA in patients with and without exfoliation. Inconsistent labelling was detected with RCA-I and CAA, particularly in patients without exfoliation (Table 2). Generally the binding of CAA and WGA was strong and that of PNA, BPA, and RCA-I moderate (Fig 2E, F), but reactivity with HPA was weak. Pretreatment with neuraminidase decreased the binding of PNA and WGA to the goblet cells.

The stromal cells of the conjunctiva were consistently labelled with RCA-I, PHA-E (Fig 2A, B), ConA, and LCA similarly in patients with and without exfoliation. The reaction was generally weak. Erythrina cristacalli agglutinin (ECA), CAA, VVA, and WGA also showed weak binding to the stroma in some specimens (Table 2). Pretreatment with neuraminidase did not alter the lectin binding pattern.

The endothelium of blood vessels showed consistent, mostly moderate reaction with RCA-I, PHA-E (Fig 2A, B), CAA, ConA (Fig 2C, D), LCA, and WGA in patients with and without exfoliation. Weak, inconsistent labelling was detected with PNA, BPA, and ECA. VVA and HPA showed also weak binding in some specimens (Table 2). The subendothelial region of blood vessels, on the other hand, reacted weakly but consistently with PHA-E, ConA, and LCA, and inconsistently with PNA in patients with and without exfoliation (Table 2). CAA and WGA reacted also weakly with this region in some specimens. Pretreatment with neuraminidase had no distinct effect upon the lectin binding to these structures.

\section{IMMUNOHISTOCHEMISTRY}

Only the myelinated stromal nerve branches in the conjunctiva were reactive with MAbs NC-1, HNK-1, or VC1.1 to the HNK-1 epitope (Fig 2G, H). The pattern and intensity of immunoreaction were identical in the conjunctiva of patients with and without exfoliation syndrome. There was no evidence of abnormal immunopositive deposits in any specimen. No reaction was detected with the control IgM antibody in any sections.

\section{Discussion}

Electron microscopic studies based on morphological criteria have detected fibrillar material resembling intraocular exfoliation fibres around blood vessels and scattered in the stroma of the bulbar and palpebral conjunctiva 
Figure 2 Labelling of the human conjunctiva with $P H A-E$, Con $A, B P A$ (A-F; lectin staining) and antibodies to the HNK-1 epitope ( $G, H$; immunoperoxidase staining). Both in a patient with exfoliation syndrome $(A ; \times 500)$ and in $a$ patient showing no exfoliation $(B ; \times 500)$, the epithelium (arrowhead) and the region of its basement membrane (double arrowhead) react moderately with $P H A-E$. The endothelial cells of the blood vessels (arrow) are strongly, and the stromal elements (str) weakly labelled. Conjunctiva from a patient with $(C ; \times 500)$ and without $(D ; \times 500)$ exfoliation syndrome show moderate binding of Con $A$ both to the epithelium (arrowhead) and to the endothelium of the blood vessels (arrow). Very weak reaction is detected within the stroma (str). BPA binds moderately to the goblet cells (arrow) of a patient with $(E ; \times 250)$ and without $(F ; \times 250)$ exfoliation syndrome. The epithelium (arrowhead), its basement membrane, the stroma (str) and the endothelium of the blood vessels (double arrowhead) show no reaction. In the conjunctiva of a patient with exfoliation, only the myelinated stromal nerve branches (arrowhead) are reactive with $M A b s$ NC-1 $(G ; \times 500)$ and $V C 1.1$ $(H ; \times 500)$. Note unstained blood vessels (arrow).
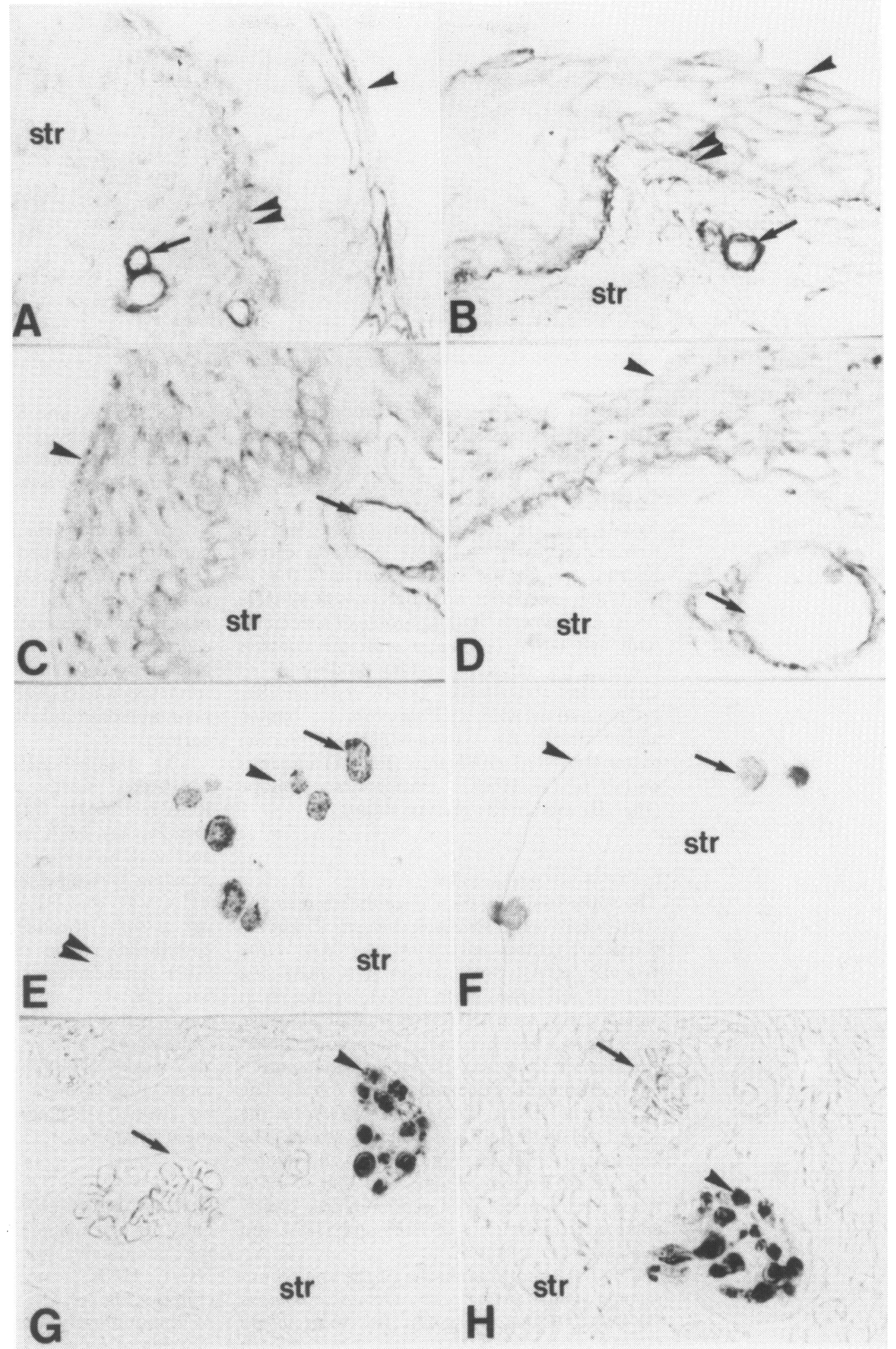

of patients with and even of those without clinical exfoliation syndrome. ${ }^{2-8}$ The occurrence of these fibres has varied from $21 \%$ to $100 \%$ in patients with clinical exfoliation syndrome, ${ }^{3-8}$ and was $35 \%$ in patients with suspected exfoliation syndrome, based on signs such as loss and dispersion of pigment from the iris pigment epithelium in the absence of overt exfoliation material on the lens capsule of either eye. ${ }^{6}$ However, variations in the degree of sharpness, width, staining properties, amount of granules, and number of adherent fibrils on the surface of these fibres have been the rule when compared with classic exfoliation fibres along the anterior chamber. ${ }^{78}$ This indicates a need for additional methods to determine the exact composition and identity of these deposits, such as the lectin histochemical and immunohistochemical approach adopted in the present study. These methods have, however, certain limitations, since both lectins and MAbs may also recognise closely 
related molecular configurations, and thus the results should be interpreted with caution. Furthermore, they may be less sensitive than electron microscopy to detect small deposits.

Intraocular exfoliation material reacts intensely with peanut (PNA), Bauhinia purpurea (BPA), Ricinus communis (RCA-I), Phaseolus vulgaris (PHA-E), and Lens culinaris (LCA) agglutinins, as well as with concanavalin A (ConA). ${ }^{15-17}$ This indicates the presence of $\alpha$-mannosyl, $\beta$-galactosyl, $N$-acetyl-D-glucosaminyl and $N$-acetyl-Dgalactosaminyl residues in glycoconjugates of exfoliation material. ${ }^{1618}$ More inconsistent binding has been detected with various other lectins. In addition, intraocular exfoliation material has been shown to react consistently with antibodies to the cell adhesion related HNK-1 carbohydrate epitope ${ }^{1719}$ containing a 3-sulphoglucuronic acid residue (Fig 1B). ${ }^{32}$

Using a panel of lectins generally reactive with exfoliation material in identically processed specimens, no abnormal lectin binding that could be attributed to exfoliation material was detected in the conjunctiva. The binding of WGA, PNA, RCA-I, and ConA to the goblet cells of patients both with and without exfoliation syndrome was identical to the results of a previous study. ${ }^{20} \mathrm{Gal}(\beta 1 \rightarrow 3)$ GalNAc-reactive lectins PNA and BPA labelled the conjunctival epithelium more often in patients with exfoliation when compared with those without it, but the significance of this finding is uncertain. The stroma was not labelled at all with PNA or BPA which rather specifically react with exfoliation material in identically processed ocular specimens. ${ }^{15} 1618$ Likewise, RCA-I, PHA-E, ConA, and LCA showed only weak reaction without abnormal deposits in the conjunctiva of patients with exfoliation syndrome. Neither were any such deposits detected by antibodies to the HNK-1 epitope. ${ }^{17}$

In addition to labelling exfoliation deposits, antibodies to the HNK-1 epitope show granular immunoreaction in the subendothelial region of a subset of iris blood vessels in eyes with exfoliation. ${ }^{17}$ Similarly, lectin binding to the subendothelial region of the iris blood vessels often resembles that of exfoliation material, although the reaction is unevenly distributed and sometimes present in control eyes without obvious classic exfoliation deposits. ${ }^{33}$ In the present study, weak but consistent binding to the subendothelial region of the conjunctival blood vessels was obtained with PHA-E, ConA, and LCA, and inconsistent binding with PNA, but no differences were noted between specimens from patients with and without exfoliation. Significantly, no labelling for the HNK-1 epitope was detected in the subendothelial region either.

Although exfoliation material, if present, may be unevenly distributed in the conjunctiva, at least some lectin or antibody binding similar to that of exfoliation material would have been expected, since the specimens were cut through. Extraocular exfoliation-like deposits have been shown to share various basement membrane components, such as heparan sulphate, chondroitin sulphate, laminin, entactin, nidogen, fibronectin, amyloid $\mathrm{P}$, and elastin with intraocular exfoliation material. ${ }^{14}$ The present findings suggest, however, that intraocular exfoliation material may differ in its carbohydrate composition from the exfoliation-like fibres often detected in the conjunctiva by electron microscopy. Taken together the fact that extraocular exfoliation-like fibres are seen in patients without overt intraocular exfoliation deposits, one might postulate that some additional, as yet unidentified, glycoconjugates are necessary to form the classic intraocular deposits of exfoliation along the anterior chamber. ${ }^{17}$ The possibility also remains that the fibrillopathy detected in extraocular sites is a different, although often parallel, pathogenetic process from intraocular exfoliation syndrome.

Supported by grants from the Finnish Eye Foundation, the Medical Research Council of the Academy of Finland, and the Emil Aaltonen Foundation.

The expert technical assistance of Mrs Marjatta Koikkalainen and Mrs Pirkko Yliharju is very much appreciated.

1 Morrison JC, Green R. Light microscopy of the exfoliation syndrome. Acta Ophthalmol (Copenh) (Suppl) 1988; 184: 5-27.

2 Ringvold A. Electron microscopy of the limbal conjunctiva in eyes with pseudo-exfoliation syndrome (PE syndrome). in eyes with pseudo-exfoliation syndro

3 Ringvold A. On the occurrence of pseudoexfoliation material in extrabulbar tissue from patients with pseudo-exfoliation syndrome of the eye. Acta Ophthalmol (Copenh) 1973 51: 411-8.

4 Layden WE, Shaffer RN. Exfoliation syndrome. Am $f$ Ophthalmol 1974; 78: 835-41.

5 Speakman JS, Ghosh M. The conjunctiva in senile lens exfoliation. Arch Ophthalmol 1976; 94: 1757-9.

6 Prince AM, Streeten BW, Ritch R, Dark AJ, Sperling M. Preclinical diagnosis of pseudoexfoliation syndrome. Arch Ophthalmol 1987; 105: 1076-82.

7 Roh YB, Ishibashi T, Ito N, Inomata $H$. Alteration of micro-fibrils on the conjunctiva of patients with exfoliation syndrome. Arch Ophthalmol 1987; 105: 978-82.

8 Streeten BW, Bookman L, Ritch R, Prince AM, Dark AJ Pseudoexfoliative fibrillopathy in the conjunctiva. A relation to elastic fibers and elastosis. Ophthalmology 1987 94: 1439-49.

9 Streeten BW, Dark AJ, Wallace RN, Li Z-Y, Hoepner JA Pseudoexfoliative fibrillopathy in the skin of patients with ocular pseudoexfoliation. Am f Ophthalmol 1990; 110 490-9.

10 Schlötzer-Schrehardt U, Küchle $M$, Naumann GOH Electron-microscopic identification of pseudoexfoliation Electron-microscopic identification of pseudoexfoliation 565-70.

11 Schlötzer-Schrehardt UM, Koca MR, Naumann GOH, Volkholz H. Pseudoexfoliation syndrome. Ocular manifestation of a systemic disorder? Arch Ophthalmol 1992; 110: 1752-6.

12 Streeten BW, Li Z-Y, Wallace RN, Eagle RC, Keshgegian AA. Pseudoexfoliative fibrillopathy in visceral organs of a patient with pseudoexfoliation syndrome. Arch Ophthalmol 1992; 110: 1757-62.

13 Ringvold A. Exfoliation syndrome. Immunological aspects. Acta Ophthalmol (Copenh) (Suppl) 1988; 184: 35-43.

14 Schlötzer-Schrehardt U, Dörfler S, Naumann GOH. Immunohistochemical localization of basement membrane components in pseudoexfoliation material of the brane components in pseudoexfoliation ma
lens capsule. Curr Eye Res 1992; 11: 343-55.

15 Streeten BW, Gibson SA, Li Z-Y. Lectin binding to exfoliative material and the ocular zonulas. Invest Ophthalmol Vi Sci 1986; 27: 1516-21.

16 Hietanen J, Tarkkanen A. Glycoconjugates in exfoliation syndrome. A lectin histochemical study of the ciliary body and lens. Acta Ophthalmol (Copenh) 1989; 67: 288-94.

17 Uusitalo $M$, Kivelä T Tarkkanen A. Immunoreactivity of exfoliation material for the cell adhesion-related HNK exfoliation material for the cell adhesion-related HNK-1 1419-23.

18 Hietanen J, Tarkkanen A, Kivelä T. Galactose-containing glycoconjugates of the ciliary body and lens. Graefes Arch glycoconjugates of the ciliary body and le
Clin Exp Ophthalmol 1994; 232: 575-83.

19 Uusitalo M. Immunohistochemical localization of chondroitin sulfate proteoglycan and tenascin in the human eye compared with the HNK-1 epitope. Graefes Arch Clin Exp Ophthalmol 1994; 232: 657-65.

20 Kawano K, Uehara F, Sameshima M, Ohba N. Application of lectins for detection of goblet cell carbohydrates of the human conjunctiva. Exp Eye Res 1984; 38: 439-47.

21 Hsu S-M, Raine L. Versatility of biotin-labelled lectins and avidin-biotin-peroxidase complex for localization of 
carbohydrate in tissue sections. $\mathcal{F}$ Histochem Cytochem 1982; 30: 157-61.

22 Kivelä T, Tarkkanen A. A lectin cytochemical study of glycoconjugates in the human retina. Cell Tissue Res 1987; glycoconjugates

23 Kivelä T. Characterization of galactose-containing glycoconjugates in the human retina: a lectin histochemical study. Curr Eye Res 1990; 12: 1195-209.

24 Leathem A, Atkins M. Lectin binding to formalin-fixed paraffin sections. $\mathcal{F}$ Clin Pathol 1983; 36: 747-50.

25 Uehara F, Muramatsu T, Sameshima M, Kawano K, Koide $\mathrm{H}$, Ohba N. Effects of neuraminidase on lectin binding sites in photoreceptor cells of monkey retina. $\mathscr{f} p n \mathcal{F}$ Ophthalmol 1985; 29: 54-62.

26 Goldstein IJ, Poretz RD. Isolation, physicochemical characterization and carbohydrate-binding specificity of lectins. In: Liener FE, Sharon N, Goldstein IJ, eds. The lectins: In: Liener FE, Sharon $\mathrm{N}$, Goldstein IJ, eds. The lectins. properties, functions and applications in biology and

27 Uusitalo M, Kivelä T, Tarkkanen A. Identification of novel element in the human eye: the inner connective tissue layer of the ciliary body characterized with antibodies to the HNK-1 epitope. Invest Ophthalmol Vis Sci 1993; 34: $2372-81$.

28 Tucker GC, Aoyama H, Lipinski M, Tursz T, Thiery JP. Identical reactivity of monoclonal antibodies HNK-1 and
NC-1: conservation in vertebrates on cells derived from the neural primordium and on some leucocytes. Cell Differ 1984; 14: 223-30.

29 Abo T, Balch CM. A differentiation antigen of human $\mathrm{NK}$ and $\mathrm{K}$ cells identified by a monoclonal antibody (HNK-1). F Immunol 1981; 127: 1024-9.

30 Arimatsu Y, Naegele JR, Barnstable J. Molecular markers of neuronal subpopulation in layers 4,5 , and 6 of cat primary visual cortex. $\mathcal{F}$ Neurosci $1987 ; 7$ : 1250-63

31 Caselitz J, Walther B, Wustrow J, Seifert G, Weber K, Osborn M. A monoclonal antibody that detects myoepithelial cells in exocrine glands, basal cells in other epithelia and basal and suprabasal cells in certain hyperplastic tissues. Virchows Arch $[A]$ 1986; 409: 725-38.

32 Margolis RK, Ripellino JA, Goossen B, Steinbrich R, Margolis RU. Occurrence of the HNK-1 epitope (3-sulfoMargolis RU. Occurrence of the HNK-1 epitope (3-sulfo-
glucuronic acid) in PC12 pheocromocytoma cells, cromaffin granule membranes, and chondroitin sulfate proteoglycans. Biochem Biophys Res Commun 1987; 145: 1142-8.

33 Hietanen J, Tarkkanen A, Kivelä T. Galactose-containing glycoconjugates of the iris, the aqueous outflow passages and the cornea in capsular glaucoma. A lectin histochemical study. Graefes Arch Clin Exp Ophthalmol (in press). 